\title{
RUMIACIÓN COGNITIVA Y AFECTO NEGATIVO COMO PREDICTORES DIFERENCIALES DE LOS SÍNTOMAS PSICOPATOLÓGICOS INTERNALIZANTES
}

\author{
COGNITIVE RUMINATION AND NEGATIVE AFFECT AS DIFFERENTIAL PREDICTORS \\ OF INTERNALIZING PSYCHOPATHOLOGICAL SYMPTOMS
}

\author{
RONALD Alberto TORO TOBAR ${ }^{1}$, Diana MARCEla ALFARO MEDina ${ }^{2}$, \\ ERIKA VIVIANA JUYÓ MOSUCA ${ }^{3}$ Y VALENTINA SANDINO SERRANO ${ }^{4}$. \\ UNIVERSIDAD CATÓLICA DE COLOMBIA
}

FECHA RECEPCIÓN: 13/03/2020 • FECHA ACEPTACIÓN: 12/09/2020

Para citar este artículo: Toro, R., Alfaro, D., Juyó, E., \& Sandino, V. ( 2020). Rumiación cognitiva y afecto negativo como predictores diferenciales de los síntomas psicopatológicos internalizantes. Psychologia, 14(2), 27-36. https://doi.org/ 10.21500/19002386.4618

\section{Resumen}

Los modelos transdiagnósticos para los problemas de ansiedad, depresión y somatización han tenido evidencia para variables como la rumiación cognitiva y el afecto negativo; sin embargo, las asociaciones diferenciales entre estas dos variables en la explicación psicopatológica requiere aún ser investigada. El objetivo fue analizar la relación entre la rumiación cognitiva según las dimensiones de la rumiación: reproche y reflexión, con el afecto negativo y los síntomas psicopatológicos internalizantes depresivos, ansiosos y psicosomáticos en adultos colombianos. Se llevó a cabo una investigación cuantitativa con un diseño transversal explicativo, en el que se definió una red estructural de relaciones entre constructos mediante un diagrama de senderos y ecuaciones estructurales. Se contó con una muestra final de 640 personas, con una edad media de 31.07 años $(D E=11.27)$. Los resultados indicaron correlaciones significativas parciales y de orden cero entre las variables transdiagnósticas y las sintomáticas internalizantes, mediante un modelo ajustado que permitió explicar en un 74 \% estas problemáticas a partir de la rumiación cognitiva y la función mediadora parcial del afecto negativo. En los síntomas depresivos y ansiosos se obtuvo que están mediados parcialmente por el afecto negativo, mientras que la medida de somatización obtuvo un coeficiente de regresión significativo con el afecto

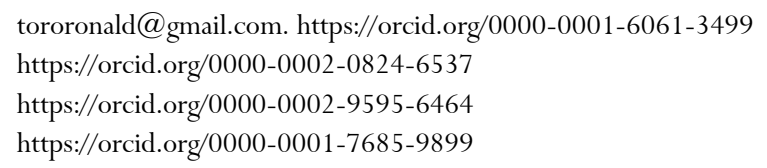


negativo como predictor independiente. Estos resultados aportan a la comprensión de la interacción de la rumiación y el afecto en la aparición de los síntomas internalizantes de manera diferencial. Se recomendó contar con una muestra clínica que ayudaría a identificar con mayor precisión la varianza explicada del modelo obtenido, lo que favorecerá los actuales tratamientos transdiagnósticos disponibles.

Palabras Clave: transdiagnóstico, rumiación, afecto negativo, psicopatología internalizante.

Abstract

The transdiagnostic models of anxiety, depression and somatization problems have had evidence for variables such as cognitive rumination and negative affect, however, the differential associations between these two variables in the psychopathological explanation, still need to be investigated. The aim was to analyze the relationship between cognitive rumination according to the two dimensions: brooding and reflection, with negative affect and depressive, anxious, and psychosomatic internalizing psychopathological symptoms. A quantitative research was carried out with an explanatory cross-sectional design in which a structural network of relationships between constructs was defined by a path diagram and structural equations. There was a final sample of 640 people with a mean age of 31.07 years $(S D=11.27)$. The results indicated significant partial and zero-order correlations between transdiagnostic and internalizing symptomatic variables, using an adjusted model that allowed explaining these problems in $74 \%$ from cognitive rumination, and the partial mediating function of negative affect. In depressive and anxious symptoms, they were partially mediated by the negative affect, while the somatization measure obtained a significant regression coefficient with the negative affect as an independent predictor. These results contribute to the understanding of the interaction of rumination and affect in the appearance of differential internalizing symptoms. It was recommended to have a clinical sample that would help to more accurately identify the explained variance of the obtained model, as well as favor the current and available transdiagnostic treatments.

Keywords: transdiagnostic, rumination, negative affect, internalizing psychopathology.

\section{Introducción}

El pensamiento negativo repetitivo es un factor transdiagnóstico de relevancia en la psicopatología, asociado a la aparición de los trastornos afectivos considerados objetivos adecuados para el tratamiento de los trastornos externalizantes. La rumiación cognitiva (CR) se caracteriza por insistir repetidamente en las causas y significados de los síntomas negativos, los sentimientos y los problemas (Nolen-Hoeksema, 1991; Watkins, 2015). A nivel transdiagnóstico, la CR está asociada a déficits en las funciones ejecutivas, en especial las habilidades metacognitivas que regulan las emociones y facilitan las conductas dirigidas hacia metas específicas (Friedman \& Miyake, 2017); si falla este proceso, se incrementa el riesgo psicopatológico dadas las falencias en los hábitos de pensamiento desadaptativo, lo que puede interrumpir los procesos, como la inhibición y la autofocalización negativa (Du Pont, et al., 2019; van Vugt \& van der Velde, 2018).

Particularmente, en los trastornos emocionales y afectivos, a nivel transdiagnóstico la $\mathrm{CR}$ se asocia al afecto negativo (NA), considerado este último como la elevada sensibilidad temperamental ante estímulos negativos, como el miedo, ansiedad, tristeza, culpa, hostilidad, insatisfacción, pesimismo, quejas somáticas y visión negativa de sí mismo (Watson, Clark, \& Tellegen, 1988). De esta manera, se ha evidenciado que en casos en los que existe la combinación entre NA y CR, aumenta la presencia de estilos cognitivos desadaptativos, actitudes disfuncionales, desesperanza, pesimismo, autocrítica, bajo dominio, dependencia, sociotropía, necesidad y neuroticismo (Nolen, Wisco, \& Lyubomirsky, 2008). La evidencia de estas vulnerabilidades cognitivas en la ansiedad, depresión, estrés y somatización resaltan la utilidad de la investigación transdiagnóstica sobre la CR 
y su relación con el NA (Brown et al., 2016; Mewton, Hobbs, Sunderland, Newby \& Andrews, 2014).

Sin embargo, no son claros todavía los mecanismos queconforman esta asociación devariables transdiagnósticas en los espectros compuestos por los síntomas depresivos, ansiosos y psicosomáticos, agrupados en psicopatologías internalizantes (Achembach et al., 2016). Se ha reportado que se debe a un pobre control cognitivo como posible moderador de la CR y los síntomas internalizantes (Snyder \& Hankin, 2016); la evidencia, por lo tanto, se orienta a la aparición de fallas en la memoria de trabajo y control emocional, lo que sugiere que se ve afectada por los niveles de estrés no explicados directamente por la CR únicamente (Snyder, Miyake \& Hankin, 2015). Esto sugiere una posible mediación del NA, que afecta las funciones ejecutivas implicadas (Smith \& Alloy, 2009). Aunque se ha verificado el papel causal de la CR en la aparición de los síntomas, la asociación con las respuestas de emocionalidad negativa no es clara, dado su posible papel mediador reportado sucintamente en estudios previos, sin tener en cuenta las formas de rumiación reproche y reflexión (Snyder \& Hankin, 2016).

En esta asociación, en términos de multifinalidad, se ha reportado una moderación significativa del consumo de alcohol en la relación reproche y síntomas internalizantes (Hilt, Armstrong \& Essex, 2015); sin embargo, ante eventos vitales negativos estresores, se ha sugerido que la aparición de la CR incrementa su capacidad predictiva si hay presencia de eventos como el rechazo en depresión o presión social por los pares en ansiedad (Nolen-Hoeksema $\&$ Watkins, 2011). En el estrés postraumático, las variables NA y CR presentan una asociación significativa diferencial con los síntomas, aunque el NA no resultó suficiente si no hacía parte de un modelo de mediación con la CR. Estos reportes sugieren que estas dos variables hacen parte de un proceso transdiagnóstico mediacional (Brown et al., 2018) en el que el estilo cognitivo rumiativo y la emocionalidad negativa tienen efectos directos e indirectos en las psicopatologías internalizantes.

De esta manera, la relación CR y NA no se ha analizado en términos mediacionalespara las psicopatologías internalizantes basadas en los síntomas depresivos, ansiosos y somáticos. En estudios previos se ha sugerido el papel mediador de la CR ante la aparición del NA y los síntomas ansiosos (Valena \& Szentagotái-Tatar, 2015), sin embargo, en el presente estudio, se hipotetiza que el NA media la relación CR y los síntomas internalizantes, dada la interferencia de las respuestas emocionales negativas en el efecto de los procesos derivados de las funciones ejecutivas (Smith \& Alloy, 2009; Snyder, Miyake \& Hankin, 2015). Adicionalmente, a través de la revisión de los efectos directos e indirectos en un modelo transdiagnóstico, en el presente estudio se buscó analizar la relación entre la rumiación cognitiva según las dimensiones de rumiación reproche y reflexión con el afecto negativo y los síntomas depresivos, ansiosos y psicosomáticos. Estos últimos no han sido analizados con detenimiento y representa un vacío en la investigación empírica hasta la fecha.

Se considera que este estudio aportará a la comprensión de las principales problemáticas en salud mental en la población adulta (Tamayo et al., 2016), dado que genera nuevas direcciones en torno a la comprensión de las elevadas tasas de comorbilidad y posibilidades de mejoras en los tratamientos futuros.

\section{Método}

Participantes

Se llevó a cabo un muestreo no probabilístico por conveniencia incidental con participantes adultos colombianos voluntarios y mayores de edad. Se estimó para un modelo predictivo un esperado de $n=472$ (error alfa del $5 \%$ ) para un análisis de regresión mediante el software GPower 3.1. El total de la muestra fue de 640 personas con una edad media de 31.07 años $(D E$ $=11.27)$. Entre los criterios de inclusión se restringió a mayores de edad con una participación voluntaria, y como exclusión, no presentar psicopatologías agudas ni estar bajo efectos de sustancias psicoactivas al momento de la evaluación psicométrica.

\section{Instrumentos}

Escalas de Depresión, Ansiedad y Estrés [DASS-21] (Antony et al., 1998). Es un instrumento de autoinforme, el cual establece una medida de la depresión, ansiedad y estrés. Está compuesto por 21 reactivos que describen estados emocionales displacenteros, en escala Likert de cuatro opciones de respuesta: de 0 ("No describe nada de lo que me pasó o sentí en la semana") hasta 3 ("Sí, esto me pasó mucho, o casi siempre”). El DASS-21 
obtuvo una adecuada consistencia en la versión en idioma español (Fonseca et al., 2010).

Escala de Afecto Positivo y Negativo [PANAS] (Watson, Clark \& Tellegen, 1988). Esta escala evalúa estados emocionales positivos y negativos. Se compone de 20 reactivos con una escala con formato tipo Likert con cinco opciones de respuesta. Se usó la versión mexicana que presenta una estructura factorial estable de dos dimensiones del instrumento, en las cuales la consistencia interna en ambas escalas está determinada por el alfa de Cronbach de .85 para el afecto positivo y .81 para el afecto negativo (Robles \& Páez, 2003).

Escala de Respuestas Rumiativas [RRS-SF] (Hervás, 2008). Es un autoinforme diseñado para medir la tendencia a rumiar como respuesta a los sentimientos de tristeza y depresión. Está compuesta por 20 reactivos evaluados mediante una escala con formato tipo Likert con cuatro opciones de respuesta $(1=$ casi nunca, y 4 = casi siempre). Se compone de dos dimensiones: Reproche y Reflexión. Los indicadores de fiabilidad de cada una de las dimensiones arrojaron un Alpha de Cronbach de .79 para Reproche y 75 Reflexión.

The Somatic Symptom Scale-8 [SSS-8] (Narrow et al., 2013). Instrumento de cribado proveniente del Patient Health Questionnaire Physical Symptoms-15 (PHQ-15) y usado como medida de referencia en el DSM $\mathrm{V}$, que identifica la sobrecarga de síntomas somáticos en los últimos 7 días. El SSS-8 está compuesto por 8 ítems con escala tipo Likert, cuyas opciones de respuestas corresponden de "No me ha molestado o nada" (0) hasta "Me ha molestado muchísimo" (4). Este test presentó una adecuada confiabilidad alfa de Cronbach de .80 (Gierk et al., 2014).

\section{Procedimiento}

La convocatoria de participación en el estudio se realizó teniendo en cuenta los criterios de inclusión y exclusión de los participantes, a quienes se les explicó su condición voluntaria y de libre retiro, el objetivo de la investigación y la firma del consentimiento informado. Las consideraciones éticas del estudio fueron previamente aprobadas por el comité de ética de la institución educativa, y se aseguró el manejo de los participantes con sintomatología clínicamente significativa, a quienes se los invitó a tratamiento psicoterapéutico gratuito en la unidad de servicios psicológicos. Una vezfirmados los documentos, se procedió a responder los instrumentos psicométricos con una duración aproximada de 15 minutos. Al terminar los test, se les mencionó a los participantes la posibilidad de acceder a los resultados del estudio, enviando un correo electrónico al equipo de investigación.

Plan de análisis de resultados. Se hizo una preparación de datos verificando los valores de normalidad univariada y multivariada, según los índices de dispersión asimétrica (-1.7 y 1.7) y curtosis (-3.5 y 3.9) (Lei \& Lomax, 2005), además del valor de curtosis multivariada con un valor recomendable inferior a 70 para modelos de senderos basados en el método de máxima verosimilitud (Rodríguez-Ayán \& Ruiz-Díaz, 2008), y la revisión de valores atípicos outliers por medio de la prueba de distancias de Mahalanobis con un punto de corte por encima $p>.001$ (Brereton, 2015). Luego, se verificó la multicolinealidad y homocedasticidad entre todas las variables; posteriormente, mediante ecuaciones estructurales, se verificó el ajuste del modelo hipotético al empírico con un análisis de senderos (path analysis), en el que se revisaron los índices de ajuste de Hu y Bentler (1999) para muestras multivariadas con diferentes distribuciones, entre ellos el Chi Square $\left(\chi^{2}\right.$, punto de corte $p>.05$, y el radio $\chi^{2} / g l$ ), el valor recomendado es $<5$. En el ajuste del modelo se tuvo en cuenta los Índices de Ajuste Global $(G F I>90)$, el Tucker-Lewis (TLI > 0.95), y el Ajuste Comparativo (CFI > 0.95), además de la Raíz del Error Cuadrático Medio de Aproximación $($ RMSEA < 0.08). Se revisaron, finalmente, los efectos directos e indirectos estandarizados para identificar la capacidad mediadora del afecto negativo en los problemas internalizantes, mediante un remuestreo usando la técnica de bootstrapping con un IC90\%. Los análisis se ejecutaron con el software AMOS-23.

Consideraciones éticas. Se siguieron las recomendaciones éticas para investigaciones con seres humanos de la Declaración de Helsinki (Asociación Médica Mundial [WMA], 2017), particularmente, lo respectivo a la participación previo conocimiento de los objetivos, el anonimato y los riesgos mínimos. Se elaboró un documento de consentimiento informado en el que aparecían los procedimientos del estudio, salvaguardando el respeto, dignidad, bienestar y los derechos de los participantes, acordes, a su vez, con las normativas del código deontológico vigentes y el comité de ética de la institución patrocinadora del proyecto. 


\section{Resultados}

Preparación de datos

Se llevó a cabo una sustitución de valores perdidos usando un método de tendencia lineal en el punto; luego, en la evaluación de valores atípicos, no se eliminaron casos, dado que los valores en la prueba de distancias de Mahalanobis estuvieron por encima de $p>$.001. En las pruebas de multicolinealidad y homocedasticidad entre las variables se obtuvieron valores favorables para los análisis multivariados posteriores $\left(r_{p}>.90, p>.01\right)$.
Estadísticos descriptivos

En la Tabla 1 se presentan los valores estadísticos obtenidos en el estudio. Se resalta que los valores medios en depresión y ansiedad estuvieron por encima del punto de corte para síntomas leves sugerido de 5 y 4 puntos, igualmente, en la escala de somatización que tiene un punto de corte de 3 puntos. Los valores de confiabilidad alfa de Cronbach de las escalas fueron favorables para las medidas transdiagnósticas y sintomáticas.

Tabla 1

Descriptivos de las variables sintomáticas y transdiagnósticas $(n=640)$

\begin{tabular}{lccccccc}
\hline \multicolumn{1}{c}{$\begin{array}{c}\text { Estadísticos / } \\
\text { Variables }\end{array}$} & $\begin{array}{c}\text { Rumiación } \\
\text { Reflexiva }\end{array}$ & $\begin{array}{c}\text { Rumiación } \\
\text { Reproche }\end{array}$ & Rumiación & $\begin{array}{c}\text { Afecto } \\
\text { Negativo }\end{array}$ & Depresión & Ansiedad & Somatización \\
\hline Media & 9.27 & 9.36 & 42.31 & 18.61 & 7.05 & 8.46 & 15.40 \\
Desviación estándar & 3.31 & 3.10 & 12.84 & 7.29 & 3.99 & 3.74 & 5.65 \\
Mínimo & 5 & 5 & 23 & 10 & 3 & 4 & 8 \\
Máximo & 20 & 20 & 89 & 50 & 24 & 23 & 37 \\
Asimetría & 0.72 & 0.98 & 0.86 & 1.21 & 1.40 & 1.13 & 0.97 \\
Curtosis & -0.08 & 0.78 & 0.50 & 1.38 & 1.90 & 0.87 & 0.64 \\
Alfa de Cronbach & .76 & .73 & .93 & .84 & .82 & .77 & .79 \\
\hline \hline
\end{tabular}

Nota: Fuente elaboración propia.

\section{Análisis correlacional y predictivo}

Se continuó con el análisis de regresión, por medio del cual se obtuvo la predicción de los síntomas internalizantes a partir de la variable rumiación cognitiva y afecto. Se encontró que las variables transdiagnósticas correlacionaron significativamente $(p<.01)$. En la Tabla 2 se presentan las correlaciones entre las medidas de rumiación y sintomáticas, en las que se controló el afecto negativo para evidenciar la posible mediación de esta variable afectiva. Se encontró que la rumiación y sus dimensiones reflexión y reproche correlacionaron elevada y significativamente con las medidas de ansiedad, depresión y somatización, además del afecto negativo. Una vez controlada esta última variable, las correlaciones disminuyeron, en particular en la variable somatización $(r=.448$ a $r=.263, p<.01)$.

Tabla 2

Correlaciones de Pearson de orden cero (izquierda abajo) y parciales controlando el afecto negativo (derecha arriba)

\begin{tabular}{lcccccc}
\hline \hline \multicolumn{1}{c}{ Variables } & 1 & 2 & 3 & 4 & 5 & 6 \\
\hline 1. Rumiación Reflexiva & - & $.554^{* *}$ & $.832^{* *}$ & $.156^{* *}$ & $.490^{* *}$ & $.436^{* *}$ \\
2. Rumiación Reproche & $.626^{* *}$ & - & $.832^{* *}$ & $.231^{* *}$ & $.552^{* *}$ & $.513^{* *}$ \\
3. Rumiación total & $.859^{* *}$ & $.864^{* *}$ & - & $.263^{* *}$ & $.635^{* *}$ & $.555^{* *}$ \\
4. Somatización & $.323^{* *}$ & $.398^{* *}$ & $.448^{* *}$ & - & $.318^{* *}$ & $.262^{* *}$ \\
5. Depresión & $.583^{* *}$ & $.642^{* *}$ & $.721^{* *}$ & $.489^{* *}$ & - & $.715^{* *}$ \\
6. Ansiedad & $.541^{* *}$ & $.613^{* *}$ & $.661^{* *}$ & $.451^{* *}$ & $.783^{* *}$ & - \\
7. Afecto negativo & $.387^{* *}$ & $.421^{* *}$ & $.486^{* *}$ & $.518^{* *}$ & $.484^{* *}$ & $.494^{* *}$ \\
\hline \hline
\end{tabular}

Nota: Fuente elaboración propia.

$* * p<.01$ 
A continuación, en un modelo de regresión lineal múltiple por pasos sucesivos, se calculó teniendo en cuenta las variables transdiagnósticas rumiación reflexiva y reproches y el afecto negativo, en un segundo bloque, como predictores, y las variables internalizantes ansiedad, depresión y somatización como las variables criterios. En ansiedad se obtuvo un coeficiente de determinación significativo $\left(A R^{2}=.466, p<.001\right)$ a partir de las variables transdiagnósticas reproche $(\beta=.375)$, reflexión $(\beta=.207)$ y afecto negativo $(\beta=.256)$. A su vez, en depresión se obtuvo un coeficiente de determinación significativo mayor $\left(A R^{2}=.503, p<.001\right)$ a partir de rumiación reproche $(\beta=.391)$, reflexión $(\beta=.253)$ y afecto negativo $(\beta=.222)$. En la variable somatización se obtuvo un coeficiente de determinación menor, pero igualmente significativo $\left(A R^{2}=.305, p<.001\right)$ a partir de las variables transdiagnósticas reproche $(\beta=.198)$ y el afecto negativo $(\beta=.421)$. La variable reflexión fue eliminada para esta última variable sintomática $(\beta=.036, p>.05)$.
Con base en estos resultados, se planteó hipotéticamente un modelo de senderos, en el que se diagramó la asociación de la rumiación cognitiva con las variables internalizantes. Los índices obtenidos no resultaron favorables según el valor del $R M S E A=.119$ (sugerido RMSEA < 0.08), aunque los demás indicadores sugerían valores dentro de los márgenes de aceptación $\left(X^{2}=70.161, D f=7, p=.000, C F I=.966, G F I=.966\right.$ y $T L I=.926)$. Se revisaron los índices de modificación del $X^{2}$ imponiendo una regresión adicional (sendero) entre el afecto negativo y la somatización (Figura 1); dado que se modificaba en 38.262 la discrepancia del modelo empírico, se resolvió comprobar un modelo diferente al hipotético inicial y se obtuvieron unos índices mucho más favorables y acordes a la teoría subyacente y las regresiones lineales previas $\left(X^{2}=6.963\right.$, $D f=6, p=.324, C F I=.999, G F I=.996, T L I=.999$ y $R M S E A=.016)$. Se logró, de esta manera, explicar en un $82 \%$ los síntomas depresivos, un $75 \%$ los ansiosos y, diferencialmente, un $36 \%$ la somatización.

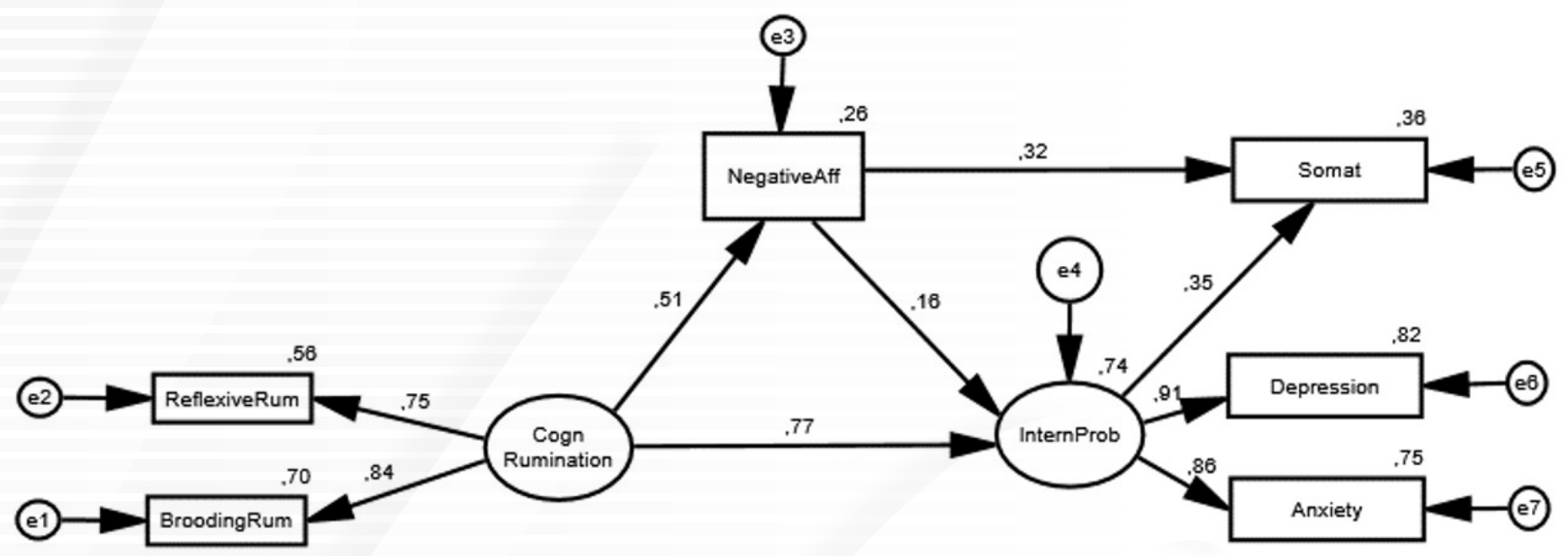

Figura 1: Modelo estructural de la asociación entre rumiación cognitiva y afecto negativo que explica las variables internalizantes en un 74\% la varianza total

Mediante una simulación de muestreo usando la técnica de bootstrapping con 2000 muestras y un sesgo corregido para intervalos de confianza del $90 \%$, se encontró que la rumiación cognitiva tiene efectos estandarizados totales significativos $\beta=.848, \beta$ IC90\% $=.806-.895, P$
$=.002)$, al igual que los efectos directos de la rumiación cognitiva $(\beta=.768, \beta$ IC90\% $=.701-.834, p=.002) \mathrm{e}$ indirectos sobre los problemas internalizantes $(\beta=.080$, $\beta$ IC90 \% $=.050-.118, p=.001)$, lo que constituye una mediación parcial del afecto negativo. 


\section{Discusión}

El objetivo de la investigación fue analizar la relación entre las dimensiones de rumiación reproche y reflexión con el afecto negativo y los síntomas internalizantes conformados por los síntomas depresivos, ansiosos y psicosomáticos. De acuerdo con los resultados obtenidos, las variables cognitivas se asociaron significativamente (CR y NA) y presentaron una favorable capacidad predictiva de las medidas sintomáticas. La rumiación cognitiva presentó efectos directos en la explicación de los síntomas depresivos y ansiosos, mediados parcialmente por el afecto negativo; mientras que en la medida de somatización se encontró un coeficiente de regresión significativo con el afecto negativo como predictor.

Estos resultados sugieren que tanto la rumiación como el afecto negativo son dos variables que tienen un impacto sobre las psicopatologías internalizantes, hallazgos que coinciden con los reportes previos a nivel cognoscitivo sobre la relación entre la rumiación y la afectividad negativa (Beck et al., 2001). Según NolenHoeksema, Wisco y Lyubomirsky (2008), las personas presentan rumiación depresiva como un intento de reducir los sentimientos negativos, que paradójicamente aumenta el afecto negativo, afecta las acciones dirigidas hacia las metas y deteriora las relaciones, lo que lleva a la aparición de problemas internalizantes. Se ha reportado que los síntomas depresivos y ansiosos comórbidos pueden ser explicados por la varianza compartida entre rumiación y preocupación (Gustavso et al., 2018), al parecer, debidos a fallas en las funciones ejecutivas, como la inflexibilidad y la memoria de trabajo (Altamirano, Miyake, \& Whitmer, 2010; Gustavson \& Miyake, 2016).

A su vez, el afecto negativo tendría un papel clave en el modelo obtenido, tal como lo sugirió Eysenck (1967), el neuroticismo como la tendencia a experimentar emociones negativas intensas en respuesta a diferentes fuentes de estrés, acompañado de una percepción generalizada de la incapacidad propia para gestionar o hacer frente a los acontecimientos difíciles. De esta manera, en la asociación la rumiación y el afecto negativo en las psicopatologías internalizantes comparten una base emocional y cognoscitiva similar, que giraría en torno a las rumiaciones (Barlow, et al., 2014), atribuida al escaso control atencional, asociado al incremento de la rumia- ción cognitiva y síntomas más graves de depresión y ansiedad (Hsu et al., 2015).

En cuanto a las dimensiones de la rumiación cognitiva, la rumiación reproche presentó una mayor explicación de la variable latente en el modelo; se logró explicar un $82 \%$ de la varianza de la depresión y un $75 \%$ en ansiedad, hallazgos que coinciden con los modelos de especificidad de síntomas según el modelo tripartito de los problemas emocionales y los hallazgos en estudios similares previos (López et al., 2012). Al parecer, la combinación entre rumiación cognitiva y los síntomas emocionales (efectos directos) estarían asociados a la experiencia de respuestas de malestar (efectos indirectos del NA) con un papel mediador parcial (efectos indirectos), tal como indicaron reportes previos sobre vulnerabilidad cognitiva a partir de contenidos esquemáticos negativos y estrés cotidiano (Ciesla, Felton \& Roberts, 2011), y el procesamiento rumiativo pre-evento y post-evento en la ansiedad social (Modini, Rapee \& Abbott, 2018), que indicaría el papel causal y sus variaciones en la respuesta emocional dada la intensidad del NA. Aunque el papel causal de la CR es relevante en el presente estudio, es necesario indagar el papel diferencial de cada componente del NA (Thomsen, 2006) en el incremento de las respuestas depresivas, ansiosas y, en particular, las somáticas, que al parecer presentan un mecanismo distinto que deberá ser analizado en posteriores estudios con mayor detenimiento.

\section{Limitaciones y conclusiones}

El estudio llevado a cabo contó con algunas limitaciones que en futuros estudios deberán ser tenidas en cuenta para mejorar el soporte de las hipótesis comprobadas. En primer lugar, la carencia de una muestra clínica que brinde validez al modelo; corregir lo anterior ayudaría a identificar con mayor precisión la varianza explicada por las variables cognitivas y afectivas del modelo revisado. En segundo lugar, las medidas internalizantes basadas en un espectro derivado de una medida psicométrica única para ansiedad y depresión; esto pudo favorecer la capacidad explicativa del modelo y pudo ser fuente de una posible autocorrelación. Se recomienda para un futuro estudio contar con medidas anexas a cada indicador sintomático del espectro internalizante.

A manera de conclusión, la asociación diferencial entre la CR y el NA en los problemas internalizantes ge- 
nera evidencias sobre la elevada capacidad predictiva de la rumiación y el papel mediador del afecto negativo, en particular, sobre la somatización, la que debería ser revisada en posteriores estudios. Se espera que los hallazgos del presente estudio tengan un impacto relevante a nivel clínico, dado que se genera una postura alternativa del análisis de la asociación entre la rumiación y el afecto negativo en las psicopatologías internalizantes, lo que permitirá el desarrollo de tratamientos transdiagnósticos más precisos y eficaces, y habilitará nuevas rutas de intervención basadas en la prevención, una vez conocidos los mecanismos de estas psicopatologías.

\section{Referencias}

Achembach, T. M., Ivanova, M. Y., Rescorla, L. A., Turner, L. V., \& Althoff, R. R. (2016). Internalizing/Externalizing Problems: Review and Recommendations for Clinical and Research Applications. Journal of the American Academy of Child and Adolescent Psychiatry, 55(8), 647-656. https://doi.org/10.1016/j. jaac.2016.05.012

Altamirano, L. J., Miyake, A., \& Whitmer, A. J. (2010). When mental inflexibility facilitates executive control: beneficial side effects of ruminative tendencies on goal maintenance. Psychological science, 21(10), 1377-1382. https://doi. org/10.1177/0956797610381505

Antony, M., Bieling, P., Cox, B., Enns, M., \& Swinson, R. (1998). Psychometric properties of the 42-item and 21-item versions of the Depression Anxiety Stress Scales (DASS) in clinical groups and a community sample. Psychological Assessment, 10(176), 176-181. https://doi.org/10.1037/1040-3590.10.2.176.

Asociación Médica Mundial [WMA] (1964). Declaración de Helsinki de la AMM- principios éticos para las investigaciones médicas en seres humanos. 1-9. Recuperado de https:/www.wma.net/es/policies-post/declaracionde-helsinki-de-la-amm-principios-eticos-para-lasinvestigaciones-medicas-en-seres-humanos.

Beck, R., Perkins, T. S., Holder, R., Robbins, M., Gray, M., \& Allison, S. H. (2001). The cognitive and emotional phenomenology of depression and anxiety: Are worry and hopelessness the cognitive correlates of NA and PA? Cognitive Therapy and Research, 25, 819827. https://doi.org/10.1023/A:1012983726272
Barlow, D., Ellard, K., Sauzer-Savala, S., Bullis, J., \& Carl, J. (2014) The origins of neuroticism. Perspectives on Psychological Science, 9(5), 481-496. https://doi. org/10.1177/1745691614544528?

Brereton, R. G. (2015). The Mahalanobis distance and its relationship to principal component scores. Journal of Chemometrics, 29(3), 143-145. https://doi. org/10.1002/cem.2692

Brown, W. J., Hetzel-Riggin, M. D., Mitchell, M. A., \& Bruce, S. E. (2018). Rumination mediates the relationship between negative affect and posttraumatic stress disorder symptoms in female interpersonal trauma survivors. Journal of Interpersonal Violence, 886260518818434, 1-22. https://doi. org/10.1177/0886260518818434

Brown, H., Meiser, R., Woods, H., \& Lester, K. (2016). Cognitive Vulnerabilities for Depression and Anxiety in Childhood: Specificity of Anxiety Sensitivity and Rumination. Behavioural and Cognitive Psychotherapy, 44(1). 30-42. https://doi.org/10.1017/ S1352465814000472.

Ciesla, J. A., Felton, J. W., \& Roberts, J. E. (2011). Testing the cognitive catalyst model of depression: Does rumination amplify the impact of cognitive diatheses in response to stress? Cognition \& Emotion, 25(8), 1349-1357. https://doi.org/10.1080/02699931.20 10.543330

Du Pont, A., Rhee, S. H., Corley, R. P., Hewitt, J. K., \& Friedman, N. P. (2019). Rumination and executive functions: Understanding cognitive vulnerability for psychopathology. Journal of Affective Disorders, 256, 550-559. https://doi.org/10.1016/j. jad.2019.06.026

Eysenck, H. J. (1967) The biological Basis of Personality. Springfield, MA: C.C, Thomas.

Fonseca, E., Paino, M., Lemos, S., \& Muñiz, J. (2010). Propiedades psicométricas de la Depression Anxiety And Stress Scale-21 (DASS-21) en universitarios españoles. Ansiedad y Estrés, 16(2), 215-226.

Friedman, N. P., \& Miyake, A. (2017). Unity and diversity of executive functions: individual differences as a window on cognitive structure. Cortex, 86, 186204. https://doi.org/10.1016/j.cortex.2016.04.023

Gierk B., Kohlmann, S., Kroenke, K., Spangenberg, L., Zenger, M., Brähler, E., \& Löwe, B. (2014). The Somatic Symptom Scale-8 (SSS-8). JAMA In- 
ternal Medicine, 174(3), 399-407. https://doi. org/10.1001.2013.12179

Gustavson, D. E., du Pont, A., Whisman, M. A., \& Miyake, A. (2018). Evidence for Transdiagnostic Repetitive Negative Thinking and Its Association with Rumination, Worry, and Depression and Anxiety Symptoms: A Commonality Analysis. Collabra. Psychology, 4(1), 13. https://doi.org/10.1525/collabra.128

Gustavson, D. E., \& Miyake, A. (2016). Trait worry is associated with difficulties in working memory updating. Cognition \& Emotion, 30(7), 1289-1303. https://doi.org/10.1080/02699931.2015.1060194

Hervás, G. (2008). Adaptación al castellano de un instrumento para evaluar el estilo rumiativo: la escala de respuestas rumiativas. Revista de Psicopatología y Psicología Clínica, 13(2), 111-121. https://doi. org/10.5944/rppc.vol.13.num.2.2008.4054.

Hilt, L. M., Armstrong, J. M., \& Essex, M. J. (2015). Rumination and moderators of multifinality: predicting internalizing symptoms and alcohol use during adolescence. Journal of Clinical Child \& Adolescent Psychology, 46(5), 746-753. https://doi.org/10.1080/1 5374416.2015 .1070354

Hsu, K. J., Beard, C., Rifkin, L., Dillon, D. G., Pizzagalli, D. A., \& Bjorgvisson, T. (2015): Transdiagnostic mechanisms in depression and anxiety: The role of rumination and attentional control. Journal of Affective Disorders, 188, 22-27. https://doi.org/10.1016/j. jad.2015.08.008

Hu, L., \& Bentler, P. M. (1999). Cutoff criteria for fit indexes in covariance structure analysis: Conventional criteria versus new alternatives. Structural Equation Modeling: A Multidisciplinary Journal, 6(1), 1-55. https://doi.org/10.1080/10705519909540118

Lei, M., \& Lomax, R. G. (2005). The effect of varying degrees of nonnormality in structural equation modeling. Structural Equation Modeling: A Multidisciplinary Journal, 12(1), 1-27. https://doi.org/10.1207/ s15328007sem1201_1

López, C. M., Felton, J. W., Driscoll, K. A., \& Kistner, J. A. (2012). Brooding rumination and internalizing symptoms in childhood: investigating symptom specificity in a multi-wave prospective study. International Journal of Cognitive Therapy, 5, 240-253. https://doi.org/10.1521/ijct.2012.5.3.240
Mewton, L., Hobbs, J., Sunderland, M., Newby, J., \& Andrews, G. (2014). Reductions in the internalising construct following internet-delivered treatment for anxiety and depression in primary care. Behaviour Research and Therapy, 63, 132-138. https://doi. org/10.1016/j.brat.2014.10.001

Modini, M., Rapee, R. M., \& Abbott, M. J. (2018). Processes and pathways mediating the experience of social anxiety and negative rumination. Behaviour Research and Therapy, 103, 24-32. https://doi. org/10.1016/j.brat.2018.01.009

Narrow, W., Clarke, D., Kuramoto, S. J., Kraemer, H. C., Kupfer, D. J., Greiner, L., \& Regier, D. A. (2013). DSM-5 field trials in the United States and Canada, part III: development and reliability testing of a cross-cutting symptom assessment for DSM-5. Archives of General Psychiatry, 170(1), 71-82. https:// doi.org/10.1176/appi.ajp.2012.12071000

Nolen-Hoeksema, S. (1991). Responses to depression and their effects on the duration of depressive episodes. Journal of Abnormal Psychology, 100(4), 569-582. https://doi.org/10.1037//0021-843x.100.4.569

Nolen-Hoeksema, S., \& Watkins, E. R. (2011). A heuristic for developing transdiagnostic models of psychopathology: Explaining multifinality and divergent trajectories. Perspectives on Psychological Science, 6(6), 589609. https://doi.org/10.1177/1745691611419672

Nolen-Hoeksema, S., Wisco, B., \& Lyubomirsky, S. (2008). Rethinking Rumination. Perspectives on Psychological Science, 3(5), 400-424. https://doi. org/10.1111/j.1745-6924.2008. 00088.x

Robles, R., \& Páez, F. (2003). Estudio sobre la traducción al español y las propiedades psicométricas de las escalas de afecto positivo y negativo (PANAS). Salud mental, 26(1), 69-75. Recuperado de http:// www.medigraphic.com/pdfs/salmen/sam-2003/ sam031h.pdf

Rodríguez-Ayán, M. N., \& Ruiz-Díaz, M. A. (2008). Atenuación de la asimetría y de la curtosis de las puntuaciones observadas mediante transformaciones de variables: Incidencia sobre la estructura factorial. $P_{s i-}$ cológica, 29, 205-227. Recuperado de https://www. uv.es/psicologica/articulos2.08/6RODRIGUEZ.pdf

Smith, J. M., \& Alloy, L. B. (2009). A roadmap to rumination: A review of the definition, assessment, and conceptualization of this multifaceted construct. 
Clinical Psychology Review, 29, 116-128. http://doi. org/10.1016/j.cpr.2008.10.003

Snyder, H. R., \& Hankin, B. L. (2016). Spiraling Out of Control. Clinical Psychological Science, 4(6), 1047-1064. https://doi.org/10.1177/2167702616633157

Snyder, H. R., Miyake, A., \& Hankin, B. L. (2015). Advancing understanding of executive function impairments and psychopathology: Bridging the gap between clinical and cognitive approaches. Frontiers in Psychology, 6, 1-24. https://doi.org/10.3389/ fpsyg. 2015.00328

Tamayo, N., Gómez-Restrepo, C., Ramírez, S., \& Rodríguez, M. N. (2016). Prevalencia de trastornos del afecto y de ansiedad en personas con condiciones crónicas. Resultado de la Encuesta Nacional de Salud Mental Colombia 2015. Revista Colombiana de Psiquiatría, 45(s1), 141-146. https://doi. org/10.1016/j.rcp.2016.06.001

Thomsen, D. K. (2006). The association between rumination and negative affect: A review. Cogni- tion \& Emotion, 20(8), 1216-1235. https://doi. org/10.1080/02699930500473533

Valena, S. P., \& Szentagotái-Tatar, A. (2015). The relationships between stress, negative affect, rumination and social anxiety. Journal of Evidence-Based Psychotherapies, 15(2), 179-189. Available at http://jebp. psychotherapy.ro/vol-xv-no-2-2015/the-relationships-between-stress-negative-affect-ruminationand-social-anxiety/

van Vugt, M. K., \& van der Velde, M. (2018). How does rumination impact cognition? A first mechanistic model. Topics in Cognitive Science, 10(1), 175-191. https://doi.org/10.1111/tops. 12318

Watkins, E. (2015). Psychological Treatment of Depressive Rumination. Current opinion in psychology, 4, 32-36. https://doi.org/10.1016/j.copsyc.2015.01.020

Watson, D., Clark, L. A., \& Tellegen, A. (1988). Development and Validation of Brief Measures of Positive and Negative Affect: The PANAS scales. Journal of Personality and Social Psychology, 54(6), 1063-1070. https://doi.org/10.1016/j.sbspro.2012.01.121 